\title{
LOS LIBROS BLANCOS DE DEFENSA DE PERÚ Y BRASIL: ASPECTOS GEOPOLÍTICOS, SEGURIDAD REGIONAL E INCIDENCIA PARA COLOMBIA ${ }^{1}$
}

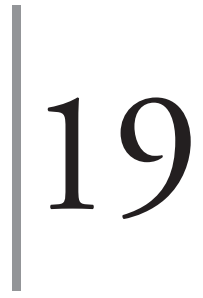

\author{
Sara Patricia Quintero Cordero \\ Manuel Ernesto Forero Garzón
}

\section{Introducción}

América Latina ha sido el área de influencia, primero del Imperio Español, el cual colonizó buena parte del continente americano. Hoy el subcontinente es la zona de influencia de los Estados Unidos, papel que le fue impuesto desde la Doctrina Monroe "América para los americanos", y ha sido desarrollada por otras doctrinas como la del Buen Vecino; y cuyas políticas esenciales se siguen implementando mediante sus planes de seguridad nacional y hemisférica, en este caso, por parte de EEUU, a través de la inversión que este país ha hecho en materia de seguridad y defensa en diversos países latinos, entre ellos Bolivia, Colombia y Perú, especialmente mediante planes para el control y eliminación del narcotráfico en el hemisferio y la lucha contra el terrorismo.

El desarrollo de este capítulo se enmarca en la teoría de Saúl Cohen, quien desarrolla la teoría del equilibrio político, para interpretar la aparición del Libro Blanco del Perú y su relación con la política geoestratégica y geopolítica imperante en el hemisferio, además, porque permitirá entender los papeles que juegan los Estados Unidos, como potencia mundial, y Brasil, como potencia regional, dentro de la configuración de la política de seguridad y defensa del Perú.

Saúl Cohen (2015) define la geopolítica como el análisis de la interacción entre las perspectivas y los entornos políticos desarrollados alrededor de la geografía y los procesos políticos que se desarrollan de modo multilateral en una determinada región. Tanto los entornos geográficos como el proceso político son altamente diná-

1 Capítulo de libro resultado del proyecto de investigación "Los libros blancos de defensa de Perú y Brasil: aspectos geopolíticos, seguridad regional e incidencia para Colombia”, aprobado por la Escuela Militar de Cadetes José María Córdova para el año 2018, y que hace parte del grupo de investigación de Ciencias Militares de la Esmic con código COL00822556, categorizado en B por Colciencias 
micos y se influyen el uno al otro de manera constante (p. 12). Asimismo, Cohen considera que las relaciones internacionales están determinadas por un sistema que contiene una jerarquía por la existencia de una escala regional en la cual existen unos campos geoestratégicos y unas regiones geopolíticas (p. 13).

En esta estructura, Cohen asume que los bloques geopolíticos son una subdivisión de los bloques geoestratégicos. Lo que determina un bloque geoestratégico son los patrones de circulación que provocan la existencia de una orientación económica y una ideología común, de manera que existe cierta homogeneidad en los flujos regionales y globales. Por otro lado, la esfera geopolítica asume que existe una cercanía geográfica entre los países que son parte de dicho bloque, además, encontramos que existen lazos históricos y culturales similares, al igual que un modo de vida semejante y unos flujos migratorios y militares que se rigen bajo las mismas necesidades (Cohen, 1991).

Cada región geopolítica está enmarcada en un escenario geoestratégico que le da un sentido dentro del marco del concierto internacional. América Latina no es la excepción y, por supuesto, cada país juega un papel dentro de dicho concierto internacional. Actualmente, la política imperante en Latinoamérica está enmarcada en la guerra contra el terrorismo y la lucha contra las drogas.

La región latinoamericana es un campo geopolítico que cumple las condiciones expresadas anteriormente por Cohen, pues existen lazos culturales e ideológicos comunes que giran alrededor de unos intereses regionales cuyo líder natural es EEUU. Este liderazgo, como se mencionó anteriormente, está marcado por los intereses panamericanos que posee Estados Unidos sobre la región y que ha implementado desde la Segunda Guerra Mundial a partir de la doctrina Monroe.

Si bien la posición hegemónica de Estados Unidos es clara, su preeminencia dentro de la política latinoamericana está siendo competida por actores locales y globales. Saúl Cohen reconoce que dicha configuración geoestratégica está en una etapa de cambio, especialmente tras el surgimiento de Brasil como potencia regional, y de Venezuela, Ecuador y Bolivia como oposiciones a las políticas tradicionalmente implementadas por la potencia del norte, además, China ha estado incursionando en la región a través de fuertes inversiones en países como Brasil y Argentina (Cohen, 2015, p. 161).

De ese modo, el objetivo de este trabajo es presentar una discusión sobre los elementos que los Libros Blancos de Defensa de Perú y Brasil podría hacer parte de una política de Seguridad y Defensa en un contexto para Colombia.

Para tal efecto, sería necesario poner la mirada en los siguientes momentos: en primer lugar analizar qué contexto geopolítico presenta Perú y Brasil en la construc- 
ción misma de su Libro Blanco; en un segundo momento especificar qué elementos relaciona Perú y Brasil dentro del Libro Blanco como estructurales para su política de seguridad y defensa que se encuentren en consonancia con el contexto presentado; y en un tercer momento observar desde los elementos analizados la posibilidad de construcción de diálogo en Colombia.

Lo anterior, radica en ver la Política planteada desde el Libro Blanco del Perú y Brasil como una herramienta útil para extraer elementos de diálogos que permitan un trabajo regional frente a las nuevas amenazas en las sociedades políticas de sus vecinos y en particular Colombia, por lo tanto, cabe destacar que este análisis tendrá un pequeño acercamiento a lo que es Perú y Brasil como Estado en su organización administrativa y militar mostrando desde su política de seguridad y defensa las orientaciones mismas en su ejercicio de poder.

\section{Contexto y Condiciones que posibilitan las Estrategias del Libro Blanco en Perú}

Perú es un país que se organizó en el 2002 durante el mandato de Alejandro Toledo bajo 26 regiones, 195 provincias y 1832 distritos.

Son 24 Departamentos como tal, a los que les suma Callao y Lima como regiones, y esta última se divide en una Área Metropolitana y Lima Provincia, la cual se ha convertido en un referente en Latinoamérica en muchos temas, como el turismo, por ejemplo, y el fortalecimiento de su economía en los últimos años. Como tal, Perú es un Estado que se propone ocupar un nuevo lugar en el mapa regional y lograr ser actor relevante en la construcción de un escenario territorial. Pero es aun consciente de que las amenazas a su propósito de Estado existen y que los problemas endógenos se han venido subsanando, y luego se encuentra que las dificultades aparecen al determinar que el crimen y los obstáculos para su horizonte son de carácter también transnacional. El Libro Blanco del Perú demuestra que este país entiende su papel regional y, que desde allí, puede lograr en esencia, una política determinada que genera un ejercicio constante de significación y retroalimentación de las amenazas a su seguridad y defensa.

Según el Banco Mundial (2018):

A lo largo de la última década, la peruana ha sido una de las economías de más rápido crecimiento en la región, con una tasa de crecimiento promedio de 5.9 por ciento en un contexto de baja inflación (promediando 2.9 por ciento). La presencia de un entorno externo favorable, políticas macroeconómicas prudentes y reformas 
estructurales en diferentes áreas crearon un escenario de alto crecimiento y baja inflación. El sólido crecimiento en empleo e ingresos redujo considerablemente las tasas de pobreza. La pobreza moderada (US\$4 por día con un PPA del 2005) cayó de 45.5 por ciento en el 2005 a 19.3 por ciento en el 2015 .

Con este panorama, el Perú apunta a fortalecerse y determinar qué podría amenazar y detener o desacelerar su ritmo de crecimiento como sociedad.

El Libro Blanco, en adelante LB, es texto y a la vez un elemento que va más allá de una idea de lo militar, es un orientador de política donde los actores en su construcción e implementación deben trabajar de manera armónica para el logro de sus objetivos. Para el caso peruano es necesario determinar el estado de cosas que enuncia el LB para su construcción o formulación.

El LB en su introducción formula que la región se caracteriza por la invisibilidad de cometidos expansionistas por parte de los países, y Perú es un país que se adhiere a las normas del derecho internacional y de allí construye su relacionamiento con sus vecinos.

El Libro Blanco de la Defensa Nacional del Perú tiene como propósito esencial dar a conocer a la ciudadanía y a los países amigos, que el Perú se guía por los principios de respeto al derecho internacional, fiel cumplimiento de los tratados, solución pacífica de controversias, respeto a la soberanía de los Estados y a las fronteras internacionales, no intervención y prohibición de la amenaza o del uso de la fuerza; en concordancia con los principios de las Cartas de la OEA y de la ONU (Ministerio de Defensa del Perú, 2005).

Por lo tanto, de entrada, puede decirse que la fuerza no es una opción primordial, ni mucho menos una garantía para la resolución de cualquier controversia desde la mirada de seguridad y defensa peruana.

En consecuencia, en un Estado de Derecho que insta al respeto de las normas fundamenta en estas su actuar y se puede, por lo tanto, en Perú, rastrear la legitimidad de su propósito en el siguiente sustento Jurídico o norma que puede convertirse en el dispositivo de acción:

Son deberes primordiales del Estado: defender la soberanía nacional; garantizar la plena vigencia de los derechos humanos; proteger a la población de las amenazas contra su seguridad; y promover el bienestar general que se fundamenta en la justicia y en el desarrollo integral y equilibrado de la Nación. Asimismo, es deber del Estado establecer y ejecutar la política de fronteras y promover la integración, particularmente latinoamericana, así como el desarrollo y la cohesión de las zonas fronterizas, en concordancia con la política exterior (Constitución Política del Perú, Art. 44). 
Cuatro criterios claves aparecen en tal enunciado normativo, a saber: la persona y en suma la población como centro de la política en el entendido de un bienestar que dice ser general. Un tema de control territorial que permita sostener la soberanía, la comprensión de un escenario común latinoamericano al cual se debe integrar y la política exterior como la herramienta para la construcción de los objetivos nacionales.

Es una mirada de adentro hacia afuera entendiendo que el entorno hace parte de los riesgos y oportunidades para el desarrollo del país.

Dado lo anterior como mandato constitucional, dicha mirada empieza a dirigirse en el sustento jurídico y político para el logro de tal norma. En el 2002, según el LB, el Acuerdo Nacional que estableció la Novena Política de Estado "Política de Seguridad Nacional", hace referencia que para su construcción se llamó a diversos líderes sociales, políticos y económicos, que vendrían a convertirse por lo tanto en acores relevantes, para generar un criterio y apuntalar una política consensuada, y define que la política de seguridad es una

Responsabilidad permanente de todos los peruanos. Tal acuerdo tiene los siguientes objetivos:

a. Fomentará la participación activa de toda la sociedad en su conjunto, en el logro de objetivos de la política de seguridad nacional.

b. Garantizará la plena operatividad de las Fuerzas Armadas orientadas a la disuasión, defensa y prevención de conflictos, así como al mantenimiento de la paz.

c. Impulsará la enseñanza de los conceptos básicos de la seguridad nacional en todos los niveles del sistema educativo nacional.

d. Fomentará la participación activa en la protección de la Antártida, el medio ambiente, el desarrollo de la Amazonía y la integración nacional.

e. Mantendrá una estrecha coordinación entre el sistema de defensa nacional y la política exterior para la definición y defensa de los intereses permanentes del Estado (Acuerdo Nacional Política de Seguridad Nacional del Perú, 2002).

Antes de continuar el rastreo propuesto, la pregunta principal a responder es ¿cuál es el concepto de seguridad con el que cuenta Perú para su dinámica política?

Vizcardo (2013) muestra un pequeño acercamiento que intenta develar cómo la protección del territorio viene desde el Imperio Inca ante las amenazas del entorno y de los conquistadores, sin embargo, es claro que el concepto actual no dista mucho de los propósitos primarios del territorio. Para tal autor, el Centro de 
Altos Estudios Nacionales, Escuela de Postgrado del Perú (CAEN-EPG), es una institución académica adscrita al sector defensa, pone la mirada en el análisis académico, estudio e investigación de los temas en seguridad, defensa y desarrollo. Allí el concepto de seguridad usado se convierte en herramienta para que los actores relevantes de la política lo utilicen como fundamento y la define como:

"el margen de protección a la que tiene derecho una determinada persona, grupo social o Estado, frente a amenazas o riesgos de diverso tipo, sean estos imaginarios o reales, naturales o sociales y que constituye un derecho inalienable del hombre, para alcanzar el bienestar y desarrollo" (p. 358).

Y el mismo CAEN-EPG plantea el siguiente concepto de Seguridad Nacional respecto de las anteriores conceptualizaciones:

"la situación en la que el Estado tiene garantizada su existencia, presencia y vigencia, así como su soberanía, independencia e integridad territorial y de su patrimonio, sus intereses nacionales, su paz y estabilidad interna, para actuar con plena autoridad y libre de toda subordinación, frente a todo tipo de amenazas" (p. 359)

Seguridad es un margen de protección que tiene por derecho un sujeto o colectivo frente a un entorno adverso, y que tal posibilidad da pie para un desarrollo sostenible y bienestar. Ahora en relación con la Seguridad Nacional la potencia del concepto radica en el fortalecimiento endógeno del Estado y sus contratantes (Vizcardo, 2013).

Son conceptos que buscan la integralidad tanto de sus actores como de los referentes, donde las amenazas pueden surgir dentro y fuera de sus fronteras y debe ser un interés de carácter nacional la preservación de la figura Estado, la cual es vista bajo los conceptos del estado liberal clásico, pero más precisa en tanto que no se habla como tal de una propuesta de régimen sino es trascendente a una política de Estado Soberano.

Por otra parte, en la ley de seguridad del Perú se hace una pesquisa sobre el concepto de Seguridad Externa e Interna que vale la pena destacar que en el ámbito interno la seguridad es "la situación en la que el estado mediante acciones de Defensa Nacional Interna garantiza al Estado mismo y a la colectividad nacional la protección contra las amenazas y conflictos que puedan surgir dentro del territorio con o sin apoyo exterior y que pongan en riesgo su supervivencia, autonomía, integridad y el logro del bienestar" (Vizcardo, 2013).

Frente a este tema es importante cómo Perú puede ver el escenario internacional como un lugar de posible apoyo para el logro de sus objetivos endógenos, y el CAEN-EPG refiere el ámbito externo así: 
"la situación en la que el Estado, mediante acciones de Defensa Nacional externa, garantiza la existencia, presencia y vigencia del Estado mismo, su independencia, soberanía, e integridad territorial y de su patrimonio, y protege a la colectividad nacional de las amenazas, riesgos y otros desafíos o presiones provenientes del exterior" (p. 359).

Visto el exterior, por lo tanto, como un posible ambiente de riesgo en particularidades, se apela a la identidad nacional y soberanía para que la defensa se convierta en el dispositivo que logre el objetivo de seguridad interna y externa; así el concepto que el autor en referencia destaca en defensa como el concepto de Defensa Nacional es: "conjunto de previsiones, decisiones y acciones que el Estado genera, adopta y ejecuta en forma integral y permanente, y que se desarrollan en los ámbitos externo e interno de la nación" (p. 360). Tales conceptos buscan una fortaleza endógena de la población, su identidad y territorio, la amenaza, por ende, tanto diversa en sus fuentes como en sus objetivos.

A partir de lo anterior, es claramente necesario apelar al LB para mirar el diálogo entre el concepto y la política. Para tal efecto es necesario observar dónde aparecen las condiciones que posibilitan el desarrollo de la política como las amenazas del sostenimiento de la paz y tranquilidad.

Una condición de carácter endógena determinada por una década de los ochenta que en su segunda mitad tuvo una crisis económica de hiperinflación, que devino en una pérdida de credibilidad en la institucionalidad por causas y efectos del desorden del gasto y desajuste fiscal, por otra parte, y comienzos de los años noventa es lo que el LB enuncia como recuperación de la institucionalidad democrática en el año 2001, en muy alta síntesis la salida de Alberto Fujimori y su política de defensa que enfrentó a grupos armados ilegales como al Movimiento Sendero Luminoso que se diluyó en su etapa final entre 1989 y 1993.

La recuperación del Perú de la crisis institucional y económica, a la par la política de seguridad que usó de la misma población civil para enfrentarse a las guerrillas, refería la necesidad de construir un ambiente de confianza tanto en la institucionalidad como entre la misma población. Era pasar la página sin olvidar.

Uno de las primeros objetivos según el LB es la modernización de las fuerzas armadas para responder a las amenazas externas y tratar de considerar que las internas, aunque son posibles, no lo son en la medida de un deterioro del interés de paz y tranquilidad nacional.

Nace en 2001 la Comisión para la Reestructuración Integral de las Fuerzas Armadas conformada por directriz del Presidente Alejandro Toledo, y una serie de actores relevantes de la sociedad civil y política que a la par de las Fuerzas Armadas 
discutieron el horizonte de la política. Tal comisión preparó un informe clave para el nuevo futuro de las instituciones armadas, donde se acusa un retroceso y la necesidad de volver a las instituciones funcionales para los ideales y retos del milenio y del Estado como tal. Así, algunas de sus consideraciones en su Informe de enero de 2002 son:

"Se trata, fundamentalmente, de situar a las Fuerzas Armadas en el entramado del Estado de Derecho, sujetas a la autoridad del Gobierno elegido legítimamente (...) las Fuerzas Armadas no intervienen ni condicionan la toma de decisiones políticas y en cambio, ejecutan la política de defensa y militar decidida por el Gobierno libremente elegido" (Comisión para la Reestructuración Integral de las Fuerzas Armadas Informe Final, 2002).

El fin primario, según el informe, es la consolidación del régimen democrático y la modernización de sus instituciones será la herramienta para tal efecto.

Por su parte, el sector armado institucional según el informe debe proponerse que jamás se creen las condiciones que subviertan la necesaria sumisión de las Fuerzas Armadas al poder Civil, por lo tanto, el control parte en el reconocimiento de roles mutuos y la instrucción suficiente para el continuo orden subordinado, donde no solo el ejecutivo garantizaría el ejercicio de lo institucionalizado, sino el congreso entraría igualmente a controlarlo. Además, surge la necesidad de un horizonte axiológico que permita un restablecimiento de la relaciones cívico-militares que como ya se nombró estaban en crisis por las dinámicas del conflicto armado, para tal acercamiento la base sería el consenso.

El horizonte axiológico propuesto se convierte desde la fecha en una posibilidad constante de retroalimentación de la relación cívico militar. Surge de la caracterización presentada como una desinstitucionalización de la fuerza por las siguientes razones expresadas en el informe:

"cuándo a través de sus respectivos comandos, en connivencia con el Gobierno dictatorial disfrazado de demócrata y en complicidad con una cúpula militar incondicional adicta al reo en cárcel Montesinos, consciente y deliberadamente se inmiscuyeron en la actividad política del país, anularon el Estado de Derecho, a la vez que montaron un aparato delictivo que usufructuó a su antojo, del Tesoro Público. Nunca antes, nuestras Fuerzas Armadas han quedado tan dañadas y cuestionadas por la opinión pública nacional y aún internacional...” (p. 4).

Por lo tanto, se presenta como necesario reinstitucionalizar, proceso que contiene los siguientes momentos: despolitización, profesionalización, fiscalización.

El análisis de este documento, en su parte inicial, podría verse como el primer paso en la transformación, tanto del horizonte institucional como de una nueva 
forma de construir las relaciones entre el Estado y sus asociados, y el Estado y sus pares que en Perú se basan en los principios democráticos.

¿Qué significa lo anterior? Que el LB es un punto más del fortalecimiento de un horizonte institucional, de una idea forjada de comprender el papel de las Fuerzas Armadas peruanas en la construcción y cohesión de las partes que integran una comunidad política. Tanto así, que en los siguientes términos solo para referencia, la continuidad del proceso muestra su estabilidad y flexibilidad en tanto que se continúa con el mismo fin y con nuevas reflexiones y normativas para desarrollar la política de Seguridad y Defensa.

Para tal efecto, el Estado Peruano se encuentra en curso de modernización, lo cual involucra a todas las dependencias de la administración pública a nivel nacional, para mejorar la gestión pública, construir un Estado democrático, descentralizado y al servicio del ciudadano (Farfán \& Núñez, 2016). El Ejército del Perú no puede ser ajeno a este proceso, por lo cual es preciso realizar las acciones necesarias, a fin de integrarse en este proceso de modernización, convirtiéndose en una directriz que acusa la necesidad de fortalecimiento del sector pertinente y surgen las siguientes aprobaciones que dan vía libre, tanto al proceso como a las medidas imperiosas para construir lo que debe llamarse como modernización, esto significa que está atada a una serie de criterios estratégicos que mostrarán no solo las amenazas, porque como más adelante se reflejará no se intenta ver a las Fuerzas Armadas como un actor reactivo, sino que se encuentran en medio de un engranaje institucional que busca un fin de Estado.

Así entonces, ante la modernización del Ejército del Perú aparecen nuevas directrices que confirman la continuidad del proceso y relaciona las que aparecen a continuación:

- $\quad$ DS 109-2012-PCM. Aprueba la estrategia para la modernización de la gestión pública el 01 de noviembre de 2012.

- DS 004-2013-PCM. Aprueba la política nacional de modernización de la gestión pública el 09 de enero de 2013.

- $\quad$ RM 125-2013-PCM. Aprueba el plan de implementación de la política nacional de modernización de la gestión pública 2013-2016 el 18 de mayo de 2013 (p. 9).

Según el Plan Bicentenario Perú hacia el 2021, en el Eje Estratégico 3: Estado y Gobernabilidad referente a la seguridad y defensa nacional se afirma que: 
Es necesario fortalecer el sistema de seguridad y defensa nacional para poder garantizar la seguridad de la nación, sin embargo, la capacidad operativa de las Fuerzas Armadas no se encuentra en un nivel óptimo debido a la obsolescencia de los equipos, la falta de programas de renovación y los bajos niveles de alistamiento, siendo necesario realizar una serie de acciones para revertir esta situación de vulnerabilidad (Farfán \& Núñez, 2016).

Lo anterior, se convierte en un elemento de reflexión, en tanto que la profesionalización y preparación de las personas de la fuerza requieren herramientas acordes a las dinámicas que las amenazas van generando, por lo tanto, hay que enfrentarlas con herramientas humanas y tecnológicas eficaces que permiten adelantarse a sí mismas y no solo concurrir ante los efectos. Así, el Plan Bicentenario es muestra fiel del desarrollo de una política en el tiempo que acusa una continua retroalimentación de su entorno.

De esta manera, se requiere mirar elementos claves del entorno que justifican los documentos relacionados y que el LB presenta para determinar derroteros en su acción.

\subsection{Entorno}

Los elementos que alimentan el proceder y gestión de la política de seguridad y defensa muestran como entorno dos niveles aspectos a referenciar. Un nivel ontológico, en tanto que define la Política de Seguridad y Defensa como vital para el mejoramiento del Índice de Desarrollo Humano, con el concurso de los países, vecinos y amigos y los organismos internacionales. Otro nivel es el sociológico, y dentro de este aparece en un primer lugar, aquel que refiere el LB de manera más precisa, y que es mencionado también en el documento de la Comisión presentada anteriormente: la globalización y con ella, se asume un determinado cambio, del cual hay que aprovechar sus ventajas y contrarrestar sus dificultades.

Para Beck (2003) la globalización significa:

Los procesos en virtud de los cuales los estados nacionales se entremezcla e imbrican mediante actores transnacionales y sus respectivas probabilidades de poder, orientaciones, identidades o entramados varios. Aquí los espacios culturales, ecológico, económicos, políticos y sociales se deben entender en una relación de mutua interdependencia... este proceso es singular por que radica en la "ramificación densidad y estabilidad de sus reciprocas redes de relaciones regionales globales empíricamente comprobables y de su autodefinición de los medios de comunicación, así como los espacios sociales y de las citadas corrientes teóricas en los planos cultural, político, militar, económico (pp. 30-31). 
Entre todas las definiciones que se encuentran sobre la globalización, esta es la más clara y concisa, en el LB del Perú es vista como una serie de procesos a los cuales hay que reconocer como evidentes, históricos y sin marcha atrás, y relaciona también sus características de manera general y amenazas. En el Libro Blanco de la Defensa Nacional aparecen como puntos y rasgos orientadores a tener en cuenta los siguientes:

1. La transnacionalización de los procesos nacionales que lleva a una convergencia hacia un cometido universal en temas estructurales como en los planos económico, social, jurídico y tecnológico, permite un espacio transnacional con múltiples amenazas como el terrorismo, narcotráfico, crimen organizado o lavado de dinero, entre otros.

2. El fortalecimiento de fuerzas locales que se resisten al proceso en pos del mantenimiento de las identidades e intereses nacionales tradicionales.

3. El surgimiento de la economía de la información y la transformación de los procesos conflictivos, incluyendo a los que implican manejo de la coerción racionalizada de Estado (conflictos interestatales).

4. Actores relevantes necesarios de ser tenidos en cuenta en los procesos de diferentes talantes, por ejemplo, ONG de tipo transnacional.

5. Una desconexión entre la realidad y los procesos institucionales internacionales (pp. 16-17).

La complejidad del proceso y, por lo tanto, sus escenarios propios de acción y efectos de sus dinámicas, muestran que cada país experimenta una serie de respuestas y acomodamientos propios o particulares que se determinan por el lugar que cada Estado ocupa en el concierto Internacional y el juego en el que puede desarrollar su inserción en un mundo globalizado.

Así pues, por ejemplo, para el mundo delictivo no es lo mismo un país productor que consumidor de estupefacientes, esto a manera de referencia de lo anteriormente comentado. La globalización se da en la medida que cada país cumple un rol en la división mundial de trabajo y esa misma define también su papel en los escenarios delictivos.

Perú hace saber en su LB que el contexto delictivo existe y que su labor es reconocer su lugar en tal escenario e identificar allí tanto las posibilidades óptimas de inserción como las amenazas tradicionales, pero además, nuevas amenazas que pueden llevar a la constitución de un complejo de seguridad en términos de Buzan en el cual la securitización ${ }^{2}$ de la agenda política de los Estados permiten dar rele-

2 Según la Escuela de Copenhague la "securitizacion” es una concepción “discursiva” de la seguridad. Un discurso político que le da importancia a una o varias amenazas donde se deben de colocar los medios y fines para contrarrestarlas, esas amenazas no son de un único actor sino de varios que por sí solos no podrían mini- 
vancia y urgencia a cierta amenazas comunes y que se complementaría en una cooperación de actores para garantizar la seguridad.

\subsection{El Papel del Perú y su Libro Blanco}

El Libro Blanco del Perú es parte del sistema internacional occidental y está orientado a ofrecer, en un marco de coherencia, confianza y cooperación, datos sobre las inversiones que el país está haciendo en materia de seguridad y defensa. El LB peruano reconoce la emergencia del marco global de intercambio de flujos de información, económicos, tecnológicos, jurídicos y políticos que están atravesando y cambiando las relaciones internacionales: la globalización y la transnacionalización. Fenómenos que han venido cambiando el modo en el cual los Estados llevan sus asuntos domésticos, pues la emergencia de grupos de presión internacionales, como las ONG, y la aparición de amenazas internacionales como las mafias globales, han llevado a cambiar las políticas internas para luchar contra estos fenómenos y han obligado a dar un viraje hacia la cooperación internacional de los Estados para mantener su propia seguridad interna. Las nuevas amenazas hemisféricas y globales han permitido que las relaciones entre dichos Estados se transformen, especialmente alrededor del terrorismo y el narcotráfico, y cuya lucha es liderada por los EEUU y la OTAN.

La influencia de Estados Unidos es evidente en el continente, o su gran mayoría, ya que se consideran afines a los preceptos ideológicos y económicos del país norteamericano. Buena parte de los países han establecido Tratados de Libre Comercio (TLC) con EEUU y tienen tratados de cooperación militar, especialmente para la lucha contra el narcotráfico. El Perú, por ejemplo, se abrió al mundo durante la década de los noventa, en el gobierno del expresidente y exdictador Alberto Fujimori, quien llevó a cabo la gran mayoría de las reformas ligadas al aperturismo económico del país y cuyo impulso se daba desde Norteamérica durante la reconfiguración de poderes después de la caída del bloque soviético.

En el 2006, durante el gobierno de Alan García, Perú firmó el Tratado de Libre Comercio (TLC) con ellos y cuyo intercambio comercial lo hace su principal socio (Cohen, 2015, p. 176), por lo cual, los lazos de cooperación se han ensanchado y robustecido. Sin duda, las alianzas determinadas por Estados Unidos con el subcontinente le dan un papel predominante en la promoción de la política exterior y la configuración de las prioridades que deben ser atendidas. Este proceso

mizarlas, por ello se hará necesario un complejo de seguridad donde interactuará a través de la cooperación para lograr la seguridad nacional y regional 
de subordinación hace parte de las configuraciones geoestratégicas que Estados Unidos han venido desarrollando en el hemisferio como parte de su propia política de seguridad.

La construcción del Libro Blanco del Perú reconoce que la seguridad se ha convertido en un tema multidimensional, dejando atrás las amenazas directas de los Estados, y asumiendo que hay temas alrededor del narcotráfico, el terrorismo y el medio ambiente que se erigen como los nuevos intereses regionales y globales (LBP, 2005, p. 13). También se parte de los principios de cooperación regional y global suscritos en la Junta Interamericana de Defensa de la OEA, a partir del capítulo VI en los artículos 27 y 28 el "principio de solidaridad continental" para justificar las alianzas estratégicas de cooperación internacional alrededor de los temas de seguridad antes descritos.

A su vez, el Libro Blanco peruano toma como principios rectores de su política internacional la democratización del continente, la cooperación y coordinación como ejes de una política multidimensional que permita hacer frente a las nuevas amenazas que presionan al continente (LBP, 2005, p. 21). Esto quiere decir que, Perú parte de la cooperación democrática, dentro del marco del respeto hacia la soberanía de los demás Estados, para desarrollar parte de sus objetivos en materia de seguridad y defensa, como: el desarrollo nacional, regional y humano y la consolidación regional de la paz y los principios democráticos (LBP, p. 21). Estos principios han sido promovidos desde Estados Unidos, por una parte, y desde la ONU, por otra.

En el entorno regional la lucha contra el narcotráfico, el terrorismo y el crimen organizado son los principales objetivos delineados por el Libro Blanco del Perú y se enfocan en el papel de las Fuerzas Militares y las políticas de seguridad y cooperación de las cuales pueden ser parte. Dentro del hemisferio, Bolivia, Colombia y Perú son tres de los países que más llaman la atención y la inversión de Estados Unidos, en cuanto son los principales países de producción de plantas de uso ilícito y, por tanto, el procesamiento de cocaína (Cohen, 2015, p. 16). Esta lucha contra el narcotráfico ha permitido que Norteamérica mantenga una inversión constante en estos países, la cual, además, está condicionada no solo a la cooperación militar sino también a la comercial e ideológica, especialmente en campos de participación internacional como la ONU.

En el entorno subregional, Perú espera participar y promover las zonas de paz en la región, la política comunitaria de paz y la promoción de la confianza en la comunidad andina de naciones, en este punto, se reconoce la autoridad del Consejo Presidencial Andino en la configuración de una política exterior y de segu- 
ridad común para la región andina (Cohen, 2015. p. 25), es decir, una geopolítica común en la región, en términos de Cohen.

Por otro lado, las relaciones vecinales con países de la Comunidad Andina como Bolivia, Ecuador, Chile y Colombia, o de cooperación amazónica como el Brasil, está marcada por los principios de seguridad, paz, gobernabilidad, defensa de los derechos humanos, defensa del medio ambiente, lucha contra la pobreza y la corrupción, el terrorismo y el narcotráfico. En este entorno geográfico vecinal, el Perú anuncia alianzas estratégicas con los países vecinos para fomentar los puntos ya mencionados, y, además, garantizar que los intereses económicos conjuntos sean satisfechos para ambas partes (Cohen, p. 30).

Hoy los temas geopolíticos que se discuten en el marco de la cooperación internacional dentro de la región latinoamericana se hacen a través de los mecanismos de cooperación como UNASUR o la OEA. La cooperación, como ya se ha dicho, gira alrededor de las amenazas que se ciernen sobre el continente en su conjunto (terrorismo, narcotráfico, amenazas medioambientales, entre otras). El Libro Blanco del Perú hace eco de dichas propuestas y es el resultado de los discursos que se han hecho protagónicos en el hemisferio, acepta y promueve los discursos multidimensionales de seguridad, los cuales no se limitan a luchar contra el narcotráfico y el terrorismo, sino que ven en los problemas medioambientales, migratorios y sociales un foco de inseguridad, no solo para el país sino para la región (LBP, 2005, p. 20). La importancia de la influencia de los Estados Unidos radica en que las decisiones alrededor de la cooperación internacional, no solo se limita al ámbito militar, sino que trasciende a los ámbitos comercial, político y social; campos en los cuales EEUU ha logrado mantener una preeminencia de sus propios intereses.

\section{Factores Geopolíticos del Libro Blanco de Defensa de Brasil}

En la evolución histórica del Estado Moderno se destacan elementos que son susceptibles en la conformación y consolidación de este: el territorio y la soberanía, para Taylor y Flint (2002) esta interrelación de variables no es suficiente para que un Estado constituya una defensa apropiada ante el poder político rival o demás Estados del escenario internacional, donde el territorio proporciona una plataforma y la soberanía una justificación que otorga legitimidad (pp. 174-177). Es a partir de esta premisa, que los Estados dentro de sus políticas públicas formulan la 
de Seguridad y Defensa, esta última, hace parte de la primera, y son condiciones necesarias para lograr niveles desarrollo. El Libro Blanco de Brasil (2012) 3 expresa la prioridad de mantener exlusiva soberanía sobre el territorio, mar territorial y espacio aéreo subyacente y no admitir la intromisión externa en sus decisiones. Además de fortalecer la cooperación con el subcontienente americano, poder de disuasión para Estados ajenos a la región y el respeto por los Principios Generales del Derecho Internacional (pp. 16-32).

Una vez consolidados sus territorios y fronteras, el territorio nacional de Brasil lo perfilan como un Estado continental y marítimo compuesto por cinco regiones: la del norte (equivale a más de la mitad del territorio y con baja densidad poblacional), el centro-oeste (la segunda en extensión territorial), el sudeste (la más poblada y desarrollada), el nordeste y la región del sur (menor extensión territorial y densamente poblada), compartiendo fronteras con nueve Estados y un territorio ultramarino de Francia, y que le representan $8.511 .965 \mathrm{~km}^{2}$, además de $4.5 \mathrm{~km}^{2} \mathrm{de}$ área marítima, siendo el quinto Estado con mayor extensión territorial del mundo. Las ventajas comparativas y competitivas del Brasil como el sector energético y minero, el agroindustrial, las extensas reservas de agua dulce y la enorme biodiversidad, entre otras, ubican al país como la sexta economía del mundo.

La dinámica del actual Sistema Internacional es influenciada por la multidimensionalidad del fenómeno de la globalización donde las unidades de este sistema se interrelacionan e integran, pero a la vez, expresan sus intenciones e intereses políticos. Dentro de esta interdependencia los Estados potencia dirigen sus políticas exteriores sobre aquellos Estados de segundo orden y tercermundistas para la obtención de recursos estratégicos que les permitan mantener sus niveles de productividad. Para Cohen, el mundo está dividido en Regiones Geoestratégicas (extensión global) y Geopolíticas ${ }^{4}$ (extensión regional), en esta última es donde nacen las potencias de segundo orden; el subcontinente de Suramérica es una Región Geopolítica y que a la vez integra la Región Geoestratégica ${ }^{5}$ del Mundo Marítimo Dependiente del Comercio, donde Brasil ha asumido la inmadurez geopolítica de la región.

Una revisión y análisis de los factores geopolíticos del Libro Blanco de Defensa de Brasil deja como resultado, que este Estado comprende la necesidad de

3 Es un documento que consolida la Estrategia de Nacional de Defensa de 2008 y revisada en 2012 y la Política de Defensa Nacional.

4 Las Américas, África, Europa marítima y el cinturón del Asia-Pacífico.

5 Otra región Geoestratégica es el Mundo Centroasiático 
la planeación, diseño y ejecución de una Política Exterior que conduzca a Brasil a ejercer funciones de Potencia Regional dentro de la Región Geopolítica de América Central, del Sur y El Caribe, para este interés estratégico, se debe desarrollar un código geopolítico. Taylor y Flint (2002) definen al código como un conjunto de supuestos estratégicos que realiza el dirigente de un Estado sobre otros Estados para orientar su política exterior con el propósito de valorar las potenciales amenazas que están más allá de las fronteras del Estado. Además, los clasifican en tres niveles: local, regional y global. El local, es una evaluación de los Estados vecinos y que tienen que ser elaborada por todos los Estados. El regional es necesario para aquellos Estados que desean proyectar poder más allá de sus fronteras, como es el caso de Brasil. Por último, unos pocos Estados contienen códigos globales que les permita expresar poder mundial (p. 99).

En el determinante geopolítico del Libro Blanco de Defensa de Brasil se identifica un código geopolítico regional incorporando zonas geográficas de importancia estratégica que ameritan el desarrollo de una política para su defensa y seguridad, estas zonas son: la Amazonía, el Atlántico Sur o Amazonía Azul y la Antártida. Estas zonas de importancia estratégica coinciden con dos áreas de valor en dicha línea de la Escuela Geopolítica Brasileña que son la aproximación a puntos marítimos importantes (desembocadura del Río Amazonas, parajes australes de Suramérica, desfiladeros atlánticos y el cabo de Buena Esperanza, sitios claves para el tráfico de petróleo con dirección al Atlántico Norte) y el área de posesión saliente de Brasil acceso más cercano entre América y Eurasia ${ }^{6}$.

Otros componentes de la escuela geopolítica de Brasil han sido la teoría orgánica del Estado que describe el espacio como fuente de poder y ampliación del territorio, el concepto de Heartland suramericano que comprende la Cuenca Amazónica y los pasos de los Andes hacia el Pacífico y una posible posesión por parte de Brasil de islas y tierras costeras en el Pacífico (Kelly y Child, 1990, pp. 115-119).

Retomando el LB de Defensa, que asume la defensa integral de las áreas estratégicas para Brasil de la Amazonía, el Atlántico Sur o Amazonía Azul y la Antártida, representando el entorno geopolítico inmediato de Suramérica, Atlántico Sur y la Costa Occidental de África. A la totalidad de la Amazonía se le denomina Pan-amazonía y es el $40 \%$ del territorio de Suramérica y de vital importancia para la humanidad al ser $20 \%$ de disponibilidad de agua dulce para el mundo, de ese

6 Otras áreas estratégicas son: abundante riqueza, población, tecnología en progreso y desarrollo de tierras internas y posición pivote como estabilizadora de las naciones de Suramérica. 
$40 \%$, el 70\% le corresponde al territorio brasileño ( 4 millones de $\mathrm{km}^{2}$ ) dentro de esta zona estratégica está la cuenca hidrográfica ${ }^{7}$ amazónica al norte del país. Para la Escuela Geopolítica Brasileña la Amazonía es el Heartland; es hábitat de numerosas especies, tierras fértiles, reservas energéticas y minerales, recorre el Río Amazonas, accede al área Andina y proyección al Pacífico.

En referencia a esta proyección, para el año 2019 se tiene planeado el inicio de la construcción del ferrocarril bioceánico que une la infraestructura de ferrocarriles de los Estados de Brasil, Bolivia y Perú, con una extensión de más de 3.500 km, y un costo aproximado de 10.000 millones de dólares, promediando una velocidad de $100 \mathrm{~km}$ por hora para trenes de carga y de $160 \mathrm{~km}$ por hora para trenes de transporte de personal, conectando el puerto de Santos (Brasil) y el puerto de Ilot en Perú. Esta iniciativa complementa el proyecto IIRSA ${ }^{8}$ del año 2000 que impulsa el proceso de integración de Suramérica, la modernización de la infraestructura y conectando los dos océanos con las carreteras oceánicas norte y sur en Perú con las carreteras de Brasil en 24 horas. Además, comunica los puertos de Santos en el Océano Pacífico y los puertos chilenos de Arica e Iquique. Estas estrategias de corredor bioceánico confirman un punto esencial del perfil de la Escuela Geopolítica Brasileña que es la proyección al Pacífico. La región del Asia-Pacífico es escenario de una Región Geoestratégica, del mayor flujo comercial del mundo, centro de poder político y recepción de recursos estratégicos necesarios para mantener la productividad de las economías de esta región.

La Amazonía para Brasil es de alta importancia geopolítica por su impacto en el desarrollo del Estado, y si es prioridad estratégica, requiere de una política para su defensa. Es aquí donde las Fuerzas Armadas consolidan la integridad territorial, ejercen disuasión e integran al Estado brasileño. Para el Ejército, la visión estratégica y de elevada prioridad es una Amazonía protegida, y está bajo la jurisdicción del Comando Militar de la Amazonía que es el de mayor extensión territorial y reúne a los Estados de Amapá, Pará, Amazonas, Roraima, Acre, Rondonia, Tocantins, Mato Grosso y Maranhao. La misión de defender y salvaguardar la Amazonía coincide con la Meta No.5 del Plan Brasil 2022 de la Secretaría de Asuntos Estratégicos que es proteger el territorio brasileńo proporcionado prioridad a la Amazonía y al centro oeste. El sector espacial de la Fuerza Aérea mantiene vigilancia de las fron-

7 El Estado brasileño alberga doce cuencas hidrográficas que permiten el transporte, interacción e integración del país con proyección a la integración de Suramérica. También destacan la mayor reserva de agua dulce subterránea del mundo (Acuífero Guaraní) y la mayor cuenca hidrográfica del mundo (el Río Amazonas).

8 Iniciativa para la Integración de la Infraestructura Suramericana; que también implica para Brasil su salida al Mar Caribe transitando por territorios de Venezuela. 
teras ${ }^{9}$ con el empleo de satélites y radares que le proporcionan una respuesta rápida ante amenazas externas, sobre todo en dicha región. De los siete Comandos Aéreos, tres tienen bajo su jurisdicción la protección de la Amazonía: los Comandos No. I (Estados de Amapá, Pará y Muranhao), VI (Tocantis y Mato Grosso) y el VII (Amazonas, Roraima, Acre y Rondonia).

En el desarrollo del factor geopolítico del Libro Blanco de Defensa, la política de fronteras define el cuerpo territorial del Estado, fija los límites y fronteras y es punto de partida para formular la defensa del espacio geográfico del Estado. Meira aborda un análisis sobre la relación entre una política de fronteras y el valor de esta para una proyección geopolítica; sostiene que, aunque el Derecho Internacional permite a los Estados de forma voluntaria someterse a sus principios, las fronteras desde la experiencia histórica se contraen o expanden de acuerdo con el poder político de los Estados que interactúan. Esto, es una condición de defensa, una frontera precisa y nítida no genera una región crítica de confrontación o de choque (LBP, 1997, pp. 17-19).

Otro interés geopolítico que aborda y analiza el LB de Brasil es el Atlántico Sur; demostrando una fuerte vinculación con el mar por factores económicos y políticos, lo que implica de forma natural ejercer una influencia sobre esta área marítima y un área estratégica para su defensa por el valor para el comercio exterior ${ }^{10}$ (LBP, 2012, p. 21). No siempre esta cualidad geopolítca ha existido en Brasil, para inicios del siglo XX con el Barón de Río Branco se consideraba al Estado como continental y no marítimo, y lo sustentaban en las amenazas de los escenarios del Río de Plata hasta la Guyana, de ahí su atraso en la Armada (Zeballos, 1974, pp. 257-277). Brasil hace parte de los 35 Estados del mundo con las mayores flotas para el comercio marítimo con 128 de pabellón nacional y 44 de pabellón extranjero para un total de 172 (Naciones Unidas, 2011, p. 49). La importancia geopolítica del Atlántico Sur o "Amazonía Azul"11 se deriva de dos puntos específicos del litoral brasileño de 7.400 km: uno es, desde el cabo Sao Roque, al Nordeste, hasta el arroyo Chuy, en el Sur, y el segundo, que va desde el cabo de Sao Roque hasta el río Oyapoque, al Norte.

9 Los Estados que comparten la Amazonia con Brasil son: Bolivia, Perú, Ecuador, Colombia, Venezuela, Surinam, Guyana, Guyana Francesa.

10 La mayor parte del comercio exterior de Brasil se moviliza por los puertos de Santos (hace parte del ferrocarril bioceánico), Río de Janeiro, Paraguaná, Recife, Salvador y Vitória, proyectándolo al África Occidental y al Atlántico Norte y Europa.

11 Lo denominan con este térmico para equipararlo con la importancia estratégica de la Amazonia. 
El primer punto específico en relación es el que conecta a Brasil con el Atlántico Sur y es donde están ubicados los principales puertos ya mencionados y de interconexión con los Estados del Cono Sur, y el segundo punto, proyecta al norte de África, a Europa, el Mar Caribe, a Centro y Norte América, y al Canal de Panamá. En suma, los dos puntos garantizan el acceso a las rutas de comercio marítimo y conexión con las Regiones Geoestratégicas descritas por Cohen, mediante los corredores estratégicos hacia el África, Europa, Centro y Norteamérica, Océano Índico, Asia y al Océano Pacífico por la parte sur del continente con el uso de los estrechos de Magallanes, Beagle y Drake que fortalecen la importancia geopolítica de la Amazonía que conecta al Pacífico con el proyecto IIRSA y el ferrocarril biooceánico.

La anterior dinámica refleja la fuerte dependencia del comercio marítimo para Brasil y el reto para su defensa. Además, para la geopolítica brasileña la Amazonía Azul es considerada una vía estratégica de comunicación con el resto del mundo. De aquí la necesidad de una defensa de sus aguas jurisdiccionales para la vigilancia, control del tráfico marítimo, seguimiento y monitoreo. La importancia geopolítica de la Amazonía se fortalece para el año 2008 y queda abordada en la Estrategia de Defensa Nacional (2008) y retomada en el LB de Defensa (2012) y que responde a dos hechos en 2008; uno nacional y otro internacional, Estados Unidos anuncia la reactivación de la Cuarta Flota ${ }^{12}$ inactiva desde la finalización de la Segunda Guerra Mundial con el propósito de combatir el narcotráfico y sus crímenes conexos. Este anuncio coincidió con el del Gobierno brasileño sobre sus reservas energéticas en aguas profundas y ultra profundas de hidrocarburos en el Atlántico Sur (Tupy y Carioca) que le permitirían dejar la dependencia externa de estos recursos. Asimismo, le permitió el desarrollo de una tecnología con la capacidad de exploración y extracción de petróleo y gas a más de 7.000 metros de profundidad.

La Amazonía Azul es un canal de comunicación con el continente blanco o Antártida (el cuarto continente más grande del mundo) con una extensión territorial de 14 millones de $\mathrm{km}^{2}$ y riquezas minerales, físicas, biológicas (que se convierten en recursos estratégicos) y adquieren cada vez más relevancia geopolítica por parte de Potencias de primer y segundo orden y de Estados de cercanía continental y de Estados de lejanía continental. La Antártida posee dos formas de protección, una

12 Esta flota hace parte del Comando Sur y tiene bajo su jurisdicción el Atlántico Sur. Es de recordar que los Estados Unidos poseen siete comandos que le permiten hacer presencia con la proyección de sus flotas navales en todas partes del mundo. Además, para ese año de reactivación el Comandante de la Cuarta Flota afirma estar en la capacidad de penetrar por la desembocadura del Río Amazonas, lo que condujo a una protesta formal y diplomática por parte del Gobierno de Brasil. 
natural y la otra es artificial, la primera es por lejanía y clima extremo pero que se ha venido reduciendo por los avances tecnológicos y la adaptación del ser humano este tipo de regiones, y la artificial, es el Trabado Antártico ${ }^{13}$ de 1961, año que entró en vigencia. Se caracteriza por ser un continente lejano, con temperaturas extremas (sus vientos son fuente de energía) y poco poblado por el ser humano y con el $70 \%$ de las reservas de agua dulce del mundo. Además, posee significativas reservas de minerales como el hierro, cromo, cobre, oro, níquel, platino, carbón e hidrocarburos. En recursos alimenticios, grandes cantidades de Krill, cangrejos, merluzas, y de organismos y microrganismos, estos dos últimos útiles para aplicaciones industriales y la investigación científica (Villamizar, 2017, pp. 18-29).

Las caracerísticas propias del continente blanco son fuentes de seguridad y desarrollo para Brasil e inicia su presencia fisica en el año de 1984 con la base de "Comandante Ferraz" para el inicio de investiaiones científicas. Esto, le proporcionará cercanía con los demás Estados con presencia allí, y conocer y compartir sus intereses políticos, comerciales y científicos. Para la protección y defensa de toda la Amanozía Azul, Brasil asigna a las Fuerzas Armadas, en menor medida para el Ejército que tiene la misión de proteger la desembocadura del Río Amazonas desde lo terrestre. A la Armada y la Fuerza Aérea sus tareas son más complejas y adscritas al Libro Blanco de Defensa y al Plan Brasil 2022 de la Secretaría de Asuntos estratégicos en las metas 2 y 4 (vigilar y proteger la totalidad del espacio aéreo brasileño ${ }^{14}$ y el aumento del poder naval para el control marítimo, negar el uso del mar y proyectar poder sobre la tierra) (2012, p. 29).

Para elevar la capacidad de defensa de la Amazonía Azul Brasil ha iniciado un programa para la actualización y modernización de su Armada con la contrucción ${ }^{15}$ de cinco submarinos; cuatro convencionales y uno nuclear, este, por transferencia de teconología francesa pero con armamentos convecionales y respetando así los términos de la Agencia Intenacional de Energía Nuclear. La Armada está constituida por nueve Comandos Navales: Río de Janeiro, Salvador, Natal, Belen, Río Grande, Ladário, Brasilia; Sao Paulo y Manaos.

13 El tratado estipula la no presencia de factores militares, cooperación científica internacional y protección de recursos, entre otros. Brasil se adhiere al tratado en el año de 1975.

14 Para la protección de los intereses geopolíticos la Fuerza Aérea de Brasil tiene desplegadas siete Comandos Aéreos: Belén, Recife, Río de Janeiro, Sao Paulo, Canoas, Brasilia y Manaos, teniendo coincidencia con algunos de los Comandos Navales de la Armada.

15 Para el aumento de su poder naval tiene planeado la construcción de 5 buques escolta, 5 buques patrulla, 1 buque de apoyo logístico, 27 buques de 500 toneladas, 1 buque de desembarco, 2 buques aeródromos y la creación de Batallones de Operaciones Ribereńas 
La Política de Defensa de Brasil que reúne a la Estrategia Nacional de Defensa y el Libro Blanco de Defensa son políticas públicas integrales que involucran no solo al estamento militar, sino que la sociedad se convierte en un actor activo en la defensa del Estado al incorporar el factor de la soberanía de la nación, la inserción económica y competitividad como fundamentos de un desarrollo pleno de Brasil. Esta visión de Seguridad y Defensa es lo que Reynolds denominó el "Interés Nacional" que lo analiza en tres apartes: la supervivencia del Estado, la generación de recursos para el bienestar de su sociedad y por último, la creación de valores para la sociedad (1977, pp. 45-59).

\subsection{Brasil y la Seguridad Regional}

El LB afirma que para Brasil la seguridad regional es esencial para la defensa por los escenarios de incertidumbre del Sistema Internacional, alimentado por el fenómeno de la globalización que ha reestructurado las relaciones de poder entre las potencias tradicionales, las emergentes, demás estados y actores. Este dinamismo necesariamente conlleva a la ampliación en temas de la agenda internacional (soberanía, drogas, terrorismo, medio ambiente, biopiratería, ilícitos internacionales, grupos armados, conflictos del futuro, entre otros). Entonces, el diálogo, el Derecho Internacional, la cooperación internacional y el multilateralismo son instrumentos válidos para perseguir el propósito de creación de las Naciones Unidas: la seguridad y paz internacionales, pero estos instrumentos no son garantía de unas relaciones entre las unidades del Sistema Internacional sin conflictos, de ahí, reafirma el LB la importancia de la Defensa Nacional (2012, pp. 33-38).

Un valor fundamental para el análisis de la seguridad regional consiste en saber cómo es vista Latinoamérica y sus ventajas comparativas; Atilo Borón demuestra que esta parte del continente americano posee las mayores reservas de petróleo, de agua dulce, minerales estratégicos, tierras fértiles, biodiversidad, especies del mundo. Además, aunque la inestabilidad política de Latinoamérica, los conflictos armados entre Estados y al interior de estos son casi nulos. Continúa afirmando que, Latinoamérica es de vital importancia estratégica pero no una prioridad para las potencias, el escenario de atención y disputa por el poder mundial ha cambiado desde hace más de dos décadas al Asia-Pacífico y como centro de atención China.

El LB presta atención a la relación entre seguridad y desarrollo, y que involucra en la defensa y la seguridad a todos los actores y componentes del Estado brasileño armonizando con los componentes de la seguridad expuestos por David al considerar que, este término- desde el fin de la Guerra Fría, ha sufrido muta- 
ciones y cada vez es más difuso, pero que debe contener tres elementos para su diseño, planeación y ejecución: involucra a toda la comunidad, la preservación de los valores de esa comunidad y la formulación de los objetivos políticos. De igual forma, esta política de seguridad puede ser ejecutada por cualquier conglomerado humano, por Organizaciones Internacionales, pero es el Estado el indicado para los temas de la seguridad de este (2008, pp. 57-92). El Libro Blanco traspasa la seguridad, y la seguridad regional hasta el nivel de la Seguridad Internacional, por todas esas amenazas tradicionales y atípicas que enfrentan las unidades o actores del Sistema Internacional. Bartolomé aborda una revisión y evolución del concepto de Seguridad Internacional; esta, deja de lado el paradigma westfaliano e introduce nuevos temas y amenazas que enfrentan no solo los Estados, sino todos los actores del Sistema Internacional, y para hacer frente a estas amenazas, se requiere con Cooperación Internacional en todos los niveles, con el fin de identificar valores e intereses en común, y poder así, dar solución de manera conjunta a esas amenazas, conduciendo el Sistema Internacional a una Comunidad de Seguridad Internacional.

Brasil identificó sus áreas de vital interés geopolitico, la forma de defenderlas, de ser incorporadas al desarrollo del Estado, la dinámica del Sistema Internacional y la complejidad e importancia en las Relaciones Internacionales de la seguridad regional e internacional. El LB de Brasil da forma multidimensional al fusionar los escenarios nacional (Derecho Interno, componentes de la sociedad y poderes nacionales del Estado), regional (diferentes mecanismos de integración, entorno geopolítico inmediato, relaciones políticas y comerciales con acotres externos a la región) e internacional (Derecho Internacional, Regiones Geoestratégicas, Organizaciones Internacionales, Seguridad y Paz Internacionales, Seguridad Regional e Internacional).

La simbiosis entre las políticas de defensa y exterior de Brasil se fundamenta en dar a conocer que el objetivo es la protección y ejercer soberanía sobre sus territorios jurisdiccionales, el poder de disuasión ante amenazas externas, el respeto y acatamiento del Derecho Internacional, la Cooperación Internacional y Latinoamérica zona de paz, pero sin prescindir de la capacidad militar del Estado brasileño. Reynolds (1977) define a la política exterior como aquellas acciones que un Estado adelanta en sus interrelaciones con los demás actores del Sistema Internacional que buscan promover el interés nacional, en concordancia con la diplomacia y la política de defensa (pp. 45-47). El perfil de la Política Exterior Brasileña vislumbra que, Brasil actúa de forma independiente de las grandes potencias, es un líder regional por naturaleza, mediador internacional, desdibuja el perfil de tercermundista, actúa 
en conjunto con la región, manifiesta el mayor liderazgo en América del Sur, tiene proyección autónoma en la política internacional, , lidera modelos de integración regional, adaptable a los cambios del Sistema Internacional, tiene participación en misiones de paz, problemáticas norte-sur, cooperación sur-sur y ampliación de los miembros permanentes del Consejo de Seguridad de las Naciones Unidas (Gomes Saravia, 2007). Una transposición del perfil de esta política con el LB, deja como resultado que, la seguridad de Brasil pasa por la seguridad regional.

En el análisis del Ambiente Estratégico del Siglo XXI que aborda el LB, la estabilidad de la región, es en este caso, factor de seguridad fortalecido desde la UNASUR $^{16}$, allí, Brasil consolida a Suramérica como una zona de paz, construye una identidad suramericana de defensa y genera consensos de cooperación regional en temas de defensa. El capítulo II del Libro en mención, detalla los aportes y participaciones del país en temas de interés para la seguridad regional, en primer lugar, promueve y hace parte del Tratado de desarme, la no proliferación y control de armas, restricciones al uso de ciertas armas convencionales y de destrucción masiva, y el Tratado de no proliferación de armas nucleares. En un segundo aparte, el Libro propone a Brasil como actor establizador en la región por sus particiaciones en misiones encomendadas por la Naciones Unidas en Colombia, Venezuela, Haití, Bolivia, entre otras.

En tercer lugar, la seguridad regional refleja esas zonas de importancia geopolitica para Brasil; la Amazonía es protegida por la Organzación del Tratado de Cooperación Amazónica (OTCA) y que impide la intención de la comunidad internacional de catalogar a esta zona geoográfica como un patrimonio de la humanidad y bloquear así los intentos de las grandes potencias que buscan los recursos estratégicos presentes allí. La defensa de la Amazonía Azul, es otro ejemplo de seguridad regional; Brasil es signatario de ZOPACAS ${ }^{17}$, Zona de Paz y Cooperación del Atlántico Sur. El continente blanco también hace parte de la seguridad regional, Brasil como parte consultiva del Tratado de la Antártida se adhiere a este en 1975. Asimismo, promueve el uso pacífico del espacio exterior.

Brasil hace presencia y participa en Organizaciones Internacionales globales, regionales y subregionales (ONU, OEA, UNASUR, MERCOUR, CELAC, G20, BRICS, Foro IBAS, Grupo BASIC, entre otros) que convocan a sus líderes y

16 Es de resaltar que los autores del escrito no se detienen en analizar la eficacia de este modelo de integración subregional, solo retoman o destacan la importancia que el Libro Blanco de Defensa hace de la UNASUR como fuente de seguridad para Brasil.

17 Creada bajo el auspicio de la ONU en 1986 y promueve la no introducción de armas nucleares al Atlántico Sur. Actualmente la componen 24 Estados. 
representantes para discutir temas políticos, sociales, económicos, comerciales, de defensa, derechos humanos, medio ambiente, desarrollo y crecimiento, y culturales que afectan la seguridad regional y la del propio Estado.

\section{El Libro Blanco de Defensa y su incidencia para Colombia}

La Política de Defensa del Brasil enfoca la integridad territorial dada por la posición geográfica y a la interacción con las demás unidades del Sistema Internacional al establecer zonas de importancia geopolítica que deben ser defendidas (Amazonía, Amazonía Azul y el Atlántico Sur con la proyección a la Antártida) que lo proyectan a Europa, al África, al Asia Pacífico, a Centro y Norte América, esto, necesariamente impone a Brasil el diseño de un código geopolítico regional que se expresa en su Política Exterior para promover el alcance del interés nacional.

Colombia comparte con Brasil ciertas similitudes en posición geográfica y proyección geopolítica; ambos Estados forman parte de la Región Geoestratégica de las Américas, la proyección para Colombia se orienta hacia el subcontinente suramericano, el Mar Caribe que lo acerca al Atlántico Norte y a Europa, a la parte occidental norte del continente africano y el Pacífico. El profesor Ricardo Esquivel (2015), analiza de forma muy detallada las Regiones Geopolíticas de mayor importancia y relevancia para Colombia; en primer lugar, la del Pacífico ${ }^{18}$ con una extensión de $390.100 \mathrm{~km}^{2}$ (sexta parte del territorio nacional y con especial interés para Panamá, Costa Rica y Ecuador) y vía de comunicación a la Región Geoestratégica de Eurasia donde China es el epicentro de poder mundial, confluencia de rutas marítimas y comerciales, y mercado para recursos estratégicos. Esta condición exige el desarrollo de un poder marítimo para Colombia. La segunda región, el Caribe, en esta región, comparte fronteras con ocho Estados ${ }^{19}$, con soberanía en $658.000 \mathrm{~km}^{2}$ catalogándolo como el Estado de mayor tamaño en el Caribe y donde confluyen las rutas marítimas con destino al Canal de Panamá, el flujo petrolero que proviene de Venezuela y las rutas de narcotráfico que se dirigen hacia el mercado de Estados Unidos. De igual forma, el profesor Esquivel demuestra que esta región es uno de los mayores caudales de agua dulce con los ríos Magdalena, Atrato, Sinú,

18 En la Plataforma Continental del Pacífico Colombiano está la Isla de Malpelo que proporciona al Estado una proyección hacia el Continente Blanco o la Antártida. La importancia geopolítica de esta zona geográfica ya ha sido analizada.

19 Panamá, Costa Rica, Nicaragua, Honduras, Jamaica, República Dominicana, Venezuela y Haití. 
Catatumbo, Zulia, Guaviare, Meta, Vichada, Arauca y Tomo. La tercera región, la suramericana, compuesta por los Andes y la Amazonía, con abundantes reservas minerales, agrícolas, agua dulce, biodiversidad, entre otros, y con recursos estratégicos para mantener el nivel de producción de las grandes potencias.

Una vez expuestas las zonas geopolíticas de vital importancia en común, similitud o posibles coincidencias de los dos Estados, se procederá rescatar del Libro Blanco de Defensa aquellos valores, factores y aportes propicios para la formulación de una política de defensa para Colombia. Para tal fin, apelamos a un perfil de defensa en concordancia con una política exterior que facilite la consecución del interés nacional. Seguido de un análisis de una política de fronteras como punto de referencia para la defensa del Estado colombiano. Por último, el papel de las Fuerzas Armadas como cuerpos de disuasión y de consecución del interés nacional.

En el LB Brasil deja en claro esa interrelación entre el interés nacional, política exterior, política de defensa y diplomacia, fundamentado en la posición geográfica de su Estado y el dinamismo de este con las demás unidades del Sistema Internacional. Antes de iniciar con la transposición del Libro Blanco sobre la realidad colombiana, es preciso mencionar que una revisión rápida a la historiografía de casi 200 años de vida republicana en Colombia, los dirigentes colombianos sacrificaron el interés personal por los intereses nacionales, definidos por Reynolds como los propósitos o metas de cada Gobierno de turno.

Inicialmente, Colombia, o sus dirigentes junto con la institucionalidad y demás actores de la sociedad, seguirán el precepto geopolítico de que todo Estado analiza sus posibles amenazas para el diseño de un código geopolítico con características de códigos locales y regionales, es decir, una evaluación de los Estados vecinos de Colombia y proyectar poder más allá de sus fronteras. Este, debe incorporar las áreas de importancia geopolítica para el país, sus zonas de influencia y proyección, que, apoyado por una política exterior dirigida por profesionales con conocimientos y experiencia en la ciencia de las Relaciones Internacionales y disciplinas afines, para la defensa del Estado colombiano. Con la noción de la defensa, todos los recursos del Estado estarán orientados a conseguir la cualidad de seguridad que incluye los factores necesarios para el desarrollo de un país.

En relación con la política de fronteras, el LB la considera como una constante que no debe sufrir modificaciones en su objetivo de definir, establecer de forma clara y nítida las fronteras mediante la firma de tratados internacionales o con los mecanismos ${ }^{20}$ alternativos de solución pacífica de controversias internacionales,

20 Otros mecanismos son: la negociación internacional, la mediación internacional, los buenos oficios, la investigación internacional y la conciliación internacional. 
como es el arbitramento internacional, el más requerido por Brasil. En la actualidad Colombia enfrenta reclamos territoriales patentes por parte de Venezuela y Nicaragua (ambos Estados con el propósito de aumentar sus zonas marítimas), este último, continúa apelando a la competencia de la Corte Internacional de Justicia, la cual fue aceptada por la Corte en el ańo 2016 ante dos reclamos que hace la República de Nicaragua. Otro ejemplo, por el cual Colombia debe consolidar sus fronteras, lo expone de forma clara el profesor Esquivel (2015); es el intento desde el 2009 de Costa Rica de ampliar su zona económica exclusiva a partir de la Isla del Coco para acceder a los bancos de atún, explotación minera y poner en duda por parte de Colombia, la posesión de la Isla de Malpelo (p. 80). Una modificación del status de Malpelo afectaría considerablemente la proyección geopolítica de Colombia sobre la Antártida.

Para el desarrollo del aparte de las Fuerzas Armadas, resulta sobresaliente cómo el Libro Blanco de Defensa asigna visiones estratégicas a cada una de las Fuerzas; para el Ejército, la elevada prioridad es la protección de la Amazonía. En la relación para la Armada, la sustenta en tres directrices: moderna (sus equipos no deben superar los 20 años de actividad), equilibrada (uso pleno del mar y aguas interiores) y balanceada (ejecutar teoría básica del poder naval). Y para la Fuerza Aérea: el fortalecimiento de la capacidad operativa, capacidad científica y tecnológica, y transformación organizacional y operativa (2012, pp. 81-152).

Estas visines estratégicas de las Fuerzas Armadas pueden convertirse es un punto de partida para Colombia en la formulación de la Política de Defensa Nacional al tener zonas geografícas de importancia geopolítica con proyección a Regiones Geoestratégicas y Geopolíticas. La Defensa para Colombia necesariamente debe pasar por los intereses geopolíticos de esta, retomando al profesor Ricardo Esquivel, Colombia actuará como potencia mediana para la protección de su integridad territorial, asegurar los recursos estratégicos e influenciar con su liderazgo en el Pacífico Occidental, los Andes, la Amazonía, el Itsmo Centroamericano, el Caribe, el Orinoco y la Guyana (2015, p. 83).

\section{Conclusiones}

Las políticas de seguridad y defensa que se desprenden de la geopolítica del hemisferio están fuertemente influenciadas por el discurso norteamericano de lucha contra el terrorismo y el narcotráfico, especialmente a partir del ataque a las torres gemelas en 2001 (Serrano Álvarez, 2018). En lo que respecta a la crisis hemisférica 
del cultivo de plantas de uso ilícito y el narcotráfico, Perú ha sido uno de los países de mayor atención por parte de Estados Unidos, pues su territorio ha sido campo de la conocida guerra contra las drogas y, además, durante la década de los noventa, campo de batalla ideológica contra Sendero Luminoso, la guerrilla de izquierda que hizo presencia en el país y que fue sometida durante el gobierno de Alberto Fujimori con apoyo estratégico y militar de los Estados Unidos.

El Libro Blanco del Perú deja entrever dicha influencia al marcar como ejes de su desarrollo los dos pilares mencionados. También se logra ver, como eje estructural, la importancia que los recursos naturales tienen para el Perú, pues su posición frente al Amazonas es estratégica y lo hace depositario de las fuentes de recursos naturales más grandes del planeta, junto a países como Colombia, Ecuador, Bolivia y Brasil.

El marco geopolítico de la región debe girar alrededor de las políticas establecidas por los Estados Unidos, sin embargo, las naciones latinoamericanas deben establecer un marco común, en el cual, desarrollen estrategias propias que ayuden a controlar y limitar los problemas regionales alrededor del cultivo de plantas de uso ilícito y narcotráfico. Este control debe salir del marco de referencia de la "guerra contra las drogas y el terrorismo" y buscar soluciones regionales que busquen controlar la crisis medioambiental, especialmente alrededor de los bosques nativos y la preservación de la Amazonía. Países como Brasil, Ecuador, Perú y Colombia deben ser los mayores depositarios de dicho cuidado, por tal razón se recomienda que asuman una estrategia geopolítica alrededor de dicha preservación. Como reconoce Perú en su Libro Blanco, las nuevas amenazas en materia de seguridad y defensa giran alrededor de las nuevas amenazas transnacionales como el narcotráfico y la pérdida de recursos naturales (LBP, 2005, p. 13). Dicha seguridad, llamada multidimensional, debe hacerse en el marco de la cooperación regional, por lo cual las políticas nacionales deben ser desbordadas y se deben asumir estas nuevas amenazas como un problema común. (Ver tambien: Fernandez-Osorio, CufiñoGutierrez, Gomez-Diaz \& Tovar-Cabrera, 2018).

Países andinos y amazónicos, como Colombia, pueden adoptar los enfoques multidimensionales usados por Perú y coordinar, desde intereses comunes, una política integradora que asuma la democracia y los derechos fundamentales, tanto de los seres humanos como de la naturaleza, como el punto de partida para construir una geopolítica que se adapte a los nuevos procesos de integración y globalización que se están dando alrededor del mundo. Perú ya reconoce esta necesidad en su política de seguridad y defensa al asumir como una realidad el cambio global alrededor de la nueva "economía de la información" (LBP, p. 10) y al identificar 
como los principales actores de dicho cambio a las ONG transnacionales, los organismos internacionales de cooperación y seguridad y a las estructuras criminales transnacionales. (Ver: Álvarez Calderón, \& Rodríguez Beltrán, 2018). Estas últimas asumidas como el principal reto de los Estados Americanos en materia de seguridad y defensa.

Por otro lado, se precisa la interrelación de la política de defensa, la política exterior y los intereses geopolíticos para la consecución del interés nacional de Brasil. Además, cómo esta dinámica favorece el estudio por parte de Colombia para formular su política de defensa por las similitudes con la posición geográfica al pertenecer a la misma Región Geopolítica de las Américas y compartir riesgos por la posesión de recursos estratégicos. Los dirigentes colombianos deben asumir la necesidad de crear un código geopolítico para el estudio y análisis de las posibles amenazas de los Estados vecinos y la proyección del poder más allá de sus fronteras. Es impresindible que la política de fronteras de Colombia las fije de forma nítida y precisa para la no generación de nuevos y pendientes reclamos territoriales.

\section{Referencias}

Álvarez Calderón, C., \& Rodríguez Beltrán, C. (2018). Ecosistemas criminales. Revista Científica General José María Córdova, 16(24), 1-30. https://doi.org/10.21830/19006586.352

Banco Mundial (2018). El Banco Mundial en Perú: Panorama General. Recuperado de http://www. bancomundial.org/es/country/peru/overview

Bartolomé, M. (2016). La guerra y la paz. Enfoque contemporáneo sobre la seguridad y la estrategia. Revista Politica y Estrategia (128), 101-134.

Beck, U. (2004). Poder y contrapoder en la era global. Nueva Economía Política Mundial. Barcelona, España: Paidós.

Beck, U. (1998). ¿Quées la globalización?: falacias del globalismo, respuestas a la globalización. Barcelona., España: Paidós.

Buzan, B. \& Hansen, L. (2009). The evolution of international security studies Inglaterra, Londres: Cambridge University Press.

Buzan, B. \& Waever, O. (2003). Regions and powers: the structure of international security. Londres, Inglaterra: Cambridge University Press.

Buzan, B, Waever, O, \& De Wilde, J. (1997). Security: A New Framework for Analysis. Ciudad, País: Lynne Rienner Publishers Inc.

Borón, A. (14 de Febrero de 2018). Análisis Geopolitico de América Latina. Recuperado el 20 de marzo de 2018 de https://www.youtube.com/watch?v=fh3k1QpF-ts

Brasil, R. d. (2012). Libro Blanco de Defensa Nacional. Ministerio de Defensa de Bradil. Recuperado el 20 de mayo de 2018. https://www.defesa.gov.br/arquivos/estado_e_defesa/livro_branco/ lbdn_esp_net.pdf 
CAEN-EPG, Planteamientos Doctrinarios. Lima. 2012.

Constitución Política del Perú, Compendio normativo, centro de Estudios Constitucionales. Recuperado de https://www.tc.gob.pe/tc/private/adjuntos/cec/publicaciones/publicacion/Compendio_Normativo.pdf

Cohen, S. (1980). Geografía y politica en un mundo dividido. Madrid, España: Ediciones Ejército.

Cohen, S. (2015). Geopolitics: the geography of international relations. Third edition. USA: Maryland. Rowman \& Littlefield.

Cohen, S. (1991). Global Geopolitical Change in the Post-Cold War Era. Annals of the Association of American Geographer. Recuperado de http://attach3.bdwm.net/attach/OAnnounce/groups/ GROUPC/GeoCultures/D888D960/DC68AD46C/M.1230483395.A/cohen.pdf

David, C. (2008). La guerra y la paz. Enfoque contemporáneo sobre la seguridad y la estrategia. Barcelona, España: Icaria.

Esquivel Triana, R. (Junio de 2015). Intereses geopoliticos de Colombia. Estudios en Seguridady Defensa, 10(19), 71-86. Recuperado el 26 de octubre de 2017 de https://www.esdeguerevistacientifica. edu.co/index.php/estudios/article/view/69/46

Farfan, M., Nuñez, E., \& Torres, A. (2016). Estrategias para la modernización del Ejército de Perú (tesis de maestría). Universidad del Pacífico, Escuela de Posgrado, Lima, Perú.

Gomes Saravia, M. (abril de 2007). Brasil y Argentina:politica externa para América Latina en tiempos recientes. Recuperado el 23 de enero de 2018 de http://revistas.usal.es/index.php/1130-2887/ article/view/2436/2485

Kelly, P. \& Child, J. (1990). Geopolitica del Cono Sur y la Antártida. Buenos Aires, Argentina: Pleamar.

Meira Mattos, C. (1997). Geopolitica y teoría de fronteras. Buenos Aires, Argentina: Círculo Militar.

Ministerio de Defensa del Perú (2005). Libro Blanco de la Defensa Nacional. Lima, Perú. Recuperado de https://www.mindef.gob.pe/libro_blanco.php

Naciones Unidas (2011). El Transporte maritimo. Conferencia de las Naciones Unidas sobre comercio y desarrollo. Recuperado el 20 de enero de 2018 de http://unctad.org/es/Docs/rmt2011_sp.pdf

Presidencia del Consejo de Ministros. (2002). Comisión para la Reestructuración Integral de las Fuerzas Armadas, Informe Final. Recuperado de http://fes-seguridadregional.org/images/ stories/docs/4111-001_g.pdf

Reynolds, P. (1977). Introducción al Estudio de las Relaciones Internacionales. Madrid, España: Tecnos.

Serrano Álvarez, J. M. (2018). El paradigma de la guerra en el siglo XX. Revista Científica General José María Córdova, 16(23), 23-42. https://doi.org/10.21830/19006586.305

Taylor, P. \& Flint, C. (2002). Geografía Política. Economía, Estado-nación y Localidad (segunda ed.) Madrid, Espańa: Trama Editorial.

Villamizar, F. (2017). Más allá de las fronteras: los horizontes geopoliticos de la antártida. Bogotá, Colombia: Ediciones Universidad Cooperativa de Colombia.

Vizcardo Benavides, R. (2013). Fundamentos conceptuales y filosóficos de la Seguridad y Defensa en el Perú. En G. Papuchi y P. Camps (eds.), Conceptos en Seguridad y Defensa en los países Iberoamericanos: Desde la óptica de sus Colegios de Defensa (pp. 357-367). Uruguay: Centro de Altos Estudios Nacionales, Ministerio de Defensa Nacional.

Zeballos, E. (1974). Diplomacia desarmada. Buenos Aires, Argentina: Universitaria. 